Nereis. Revista Iberoamericana Interdisciplinar de Métodos, Modelización y Simulación

12

185-193

Universidad Católica de
Valencia San Vicente Mártir
Valencia
(España)

ISSN $1888-8550$

\title{
Cálculo de la flecha en la lemniscata de Bernoulli. Aplicación a curvas de transferencia ferroviarias
}

\author{
Calculation of the deflection in the lemniscate of Bernoulli. \\ Application to railway transfer curves
}

Fecha de recepción y aceptación: 21 de noviembre de 2019, 21 de abril de 2020

DOI: $10.46583 /$ nereis_2020.12.523

\section{J. L. González-Santander ${ }^{1^{*}}$}

${ }^{1}$ Departamento de Matemáticas. Universidad de Oviedo.

${ }^{*}$ Correspondencia: Universidad de Oviedo. Departamento de Matemáticas. Facultad de Ciencias. Avda. de Calvo Sotelo s/n 33007 Oviedo. España.

E-mail: gonzalezmarjuan@uniovi.es

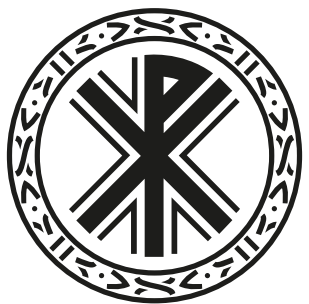

\section{RESUMEN}

Se deducen unas expresiones analíticas para calcular la flecha entre una cuerda y una curva lemniscata de Bernoulli. El interés de estas expresiones está en que, en los trazados ferroviarios, las lemniscatas se utilizan como curvas de transferencia entre un tramo recto y otro circular. Es más, según la normativa, la flecha que separa un vagón de la vía no debe exceder una determinada cantidad, por lo que las expresiones deducidas son de gran utilidad en las comprobaciones rutinarias de los trazados ferroviarios.

PALABRAS CLAVE: lemniscata de Bernoulli, curvas de transferencia.

\section{ABSTRACT}

Analytical expressions are deduced to calculate the deflection between a chord and a lemniscate curve. In railways, the interest of these expressions is that lemniscates are used as transfer curves between a straight and a circular section. Moreover, according to the regulations, the deflection that separates a wagon from the track must not exceed a certain amount, so that the expressions deduced are very useful in routine checks of railway tracks.

KEYWORDS: lemniscate, transfer curves. 


\section{INTRODUCCIÓN}

En el diseño de las vías ferroviarias se acostumbra a utilizar como trazado de transferencia, entre un tramo recto y otro de curvatura constante, diversos tipos de curvas como las parábolas cúbicas, la clotoide o la lemniscata de Bernoulli [1]. En el presente artículo consideraremos la lemniscata como curva de transferencia. En la figura 1 se ha representado el trazado de la vía centrando un sistema de coordenadas cartesiano en el punto donde el tramo recto empieza a curvarse según una lemniscata.

Si $P=\left(x_{P}, y_{P}\right)$ es un punto cualquiera de la curva, tenemos que

$$
\tan \alpha=\frac{y_{P}}{x_{P}},
$$

donde suponemos, sin pérdida de generalidad que $x_{P}>y_{P}$. En caso contrario, cámbiese $x_{P}$ por $y_{P}$, dado que el problema es simétrico respecto a si el tren se desvía a la derecha o la izquierda según el sentido de marcha.

Una de las comprobaciones rutinarias de estos trazados es saber si satisfacen la normativa de seguridad, la cual, entre otras cosas, consiste en que la distancia máxima entre una cuerda y la curva, denominada flecha (véase $f$ en la figura 3), no exceda una determinada cantidad. Por esta razón, el objetivo de este artículo consiste en obtener una expresión analítica que permita calcular la flecha generada por dos puntos cualesquiera de una lemniscata.

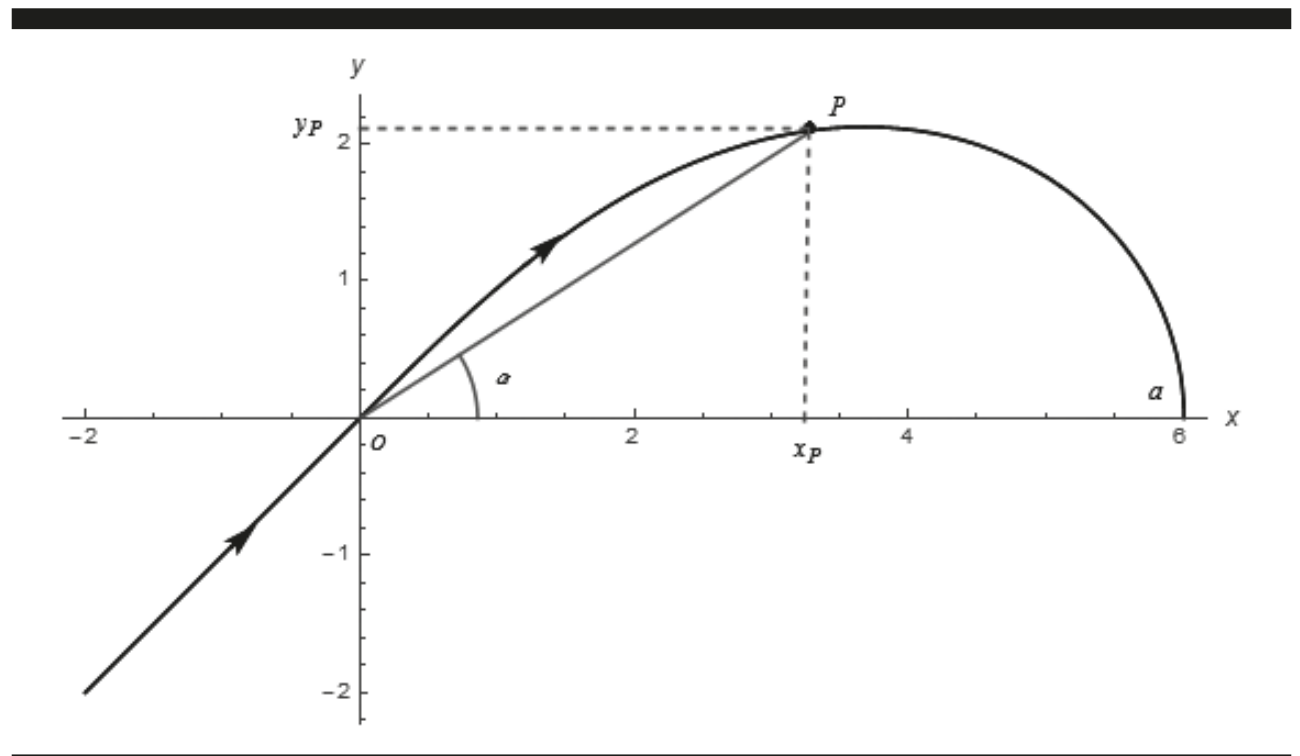

Fig. 1. Trayectoria descrita por el tren.

Dado que tenemos que tratar con la curva lemniscata, en primer lugar procedemos a hacer un repaso de las diferentes expresiones de esta curva y su derivada en coordenadas paramétricas. Con estas expresiones podremos calcular la flecha entre dos puntos cualesquiera de dicha curva. 


\section{LA LEMNISCATA DE BERNOULLI}

La expresión de la curva lemniscata en coordenadas polares viene dada por [2]:

$$
r(\theta)=a \sqrt{\cos 2 \theta}
$$

Obsérvese que $r(0)=a$ y $r(\pi / 4)=0$, en consonancia con la figura 1. En coordenadas cartesianas, (2) se escribe de forma implícita como:

$$
\left(x^{2}+y^{2}\right)^{2}=a^{2}\left(x^{2}-y^{2}\right)
$$

y en forma explícita como:

$$
y= \pm \sqrt{\frac{a}{2} \sqrt{a^{2}+8 x^{2}}-x^{2}-\frac{a^{2}}{2}} .
$$

Notése que según (4) la curva lemniscata es una función par, $y(-x)=y(x)$, con dos ramas, una con el signo '+' y otra con el signo '-'. Asimismo, obsérvese que si nos dan un punto de la lemniscata en coordenadas cartesianas, $P=\left(x_{P} y_{P}\right)$, a partir de (3), podemos calcular el parámetro $a$ de la lemniscata,

$$
a=\frac{x_{P}^{2}+y_{P}^{2}}{\sqrt{x_{P}^{2}-y_{P}^{2}}}
$$

Cabe destacar que como estamos considerando $x_{P}>y_{P}$, tenemos que $a>0$.

Si tomamos como parámetro el ángulo polar $\theta$, a partir de (2) es inmediata la siguiente representación paramétrica:

$$
\left\{\begin{array}{l}
x(\theta)=a \cos \theta \sqrt{\cos 2 \theta} \\
y(\theta)=a \sin \theta \sqrt{\cos 2 \theta}
\end{array}\right.
$$

Con las identidades trigonométricas:

$$
\cos \theta=\frac{1}{\sqrt{1+\tan ^{2} \theta}}, \quad \sin \theta=\frac{\tan \theta}{\sqrt{1+\tan ^{2} \theta}}, \quad \cos 2 \theta=\frac{1-\tan ^{2} \theta}{1+\tan ^{2} \theta},
$$


y realizando el cambio $\tan \theta=\cos \varphi$, llegamos a la representación paramétrica:

$$
\left\{\begin{array}{l}
x(\varphi)=\frac{a \sin \varphi}{1+\cos ^{2} \varphi}, \\
y(\varphi)=\frac{a \cos \varphi \sin \varphi}{1+\cos ^{2} \varphi} .
\end{array}\right.
$$

Finalmente, aplicando el cambio $t=\tan (\varphi / 2)$, llegamos a:

$$
\left\{\begin{array}{l}
x(t)=\frac{a t\left(1+t^{2}\right)}{1+t^{4}}, \\
y(t)=\frac{a t\left(1-t^{2}\right)}{1+t^{4}}
\end{array}\right.
$$

Observemos que cuando $t=0$, la curva se encuentra en el origen $(0,0)$, y cuando $t=1$, la curva se encuentra en el extremo $(a, 0)$, por lo que consideraremos el tramo de curva para el cual $t \in[0,1]$, es decir, la parte de curva que se halla sobre el primer cuadrante.

Derivando en (9) con respecto a $t$, resulta que,

$$
\frac{d y}{d x}=\frac{d y / d t}{d x / d t}=\frac{1-3 t^{2}-3 t^{4}+t^{6}}{1+3 t^{2}-3 t^{4}-t^{6}}
$$

Obsérvese que en el origen, es decir, $\forall t=0$, la pendiente de la curva es 1 , es decir, $y^{\prime}(0)=1$. Asimismo, la pendiente $\forall t \rightarrow 1^{-}$, la pendiente resulta ser $-\infty$, es decir, $\lim _{x \rightarrow a^{-}} y^{\prime}(x)=-\infty$.

\section{CÁLCULO DE LA POSICIÓN DE LA CABECERA DEL VAGÓN}

En la figura 2 se ha representado la posición de un vagón de longitud $L$ sobre la curva de transferencia. Conocida la posición de la cola del vagón (punto $A$ ), se trata de calcular la posición de la cabecera (punto $B$ ). Aplicando el teorema del coseno al triángulo $O A B$ de la figura 2 , tenemos que

$$
L^{2}=r_{1}^{2}+r_{2}^{2}-2 r_{1} r_{2} \cos \left(\theta_{1}-\theta_{2}\right)
$$

Teniendo en cuenta la expresión en polares de la curva lemniscata (2) y desarrollando el coseno de la diferencia, (11) se convierte en

$$
\left(\frac{L^{2}}{a^{2}}-\cos 2 \theta_{1}-\cos 2 \theta_{2}\right)^{2}=4 \cos 2 \theta_{1} \cos 2 \theta_{2}\left(\cos \theta_{1} \cos \theta_{2}+\sin \theta_{1}+\sin \theta_{2}\right)^{2}
$$


Recordemos que nuestro objetivo es calcular la posición de la cabecera del vagón a partir de la posición de su cola, por lo que consideraremos como incógnita a resolver $x=\cos 2 \theta_{2}$. De esta forma, operando en (12) y haciendo uso de las fórmulas trigonométricas del ángulo mitad del seno y el coseno, llegamos a la siguiente expresión:

$$
\left[\left(\frac{L^{2}}{a^{2}}-\cos 2 \theta_{1}-x\right)^{2}-2 x \cos \theta_{1}\left(x \cos 2 \theta_{1}+1\right)\right]^{2}=\sin ^{2} 4 \theta_{1}\left(1-x^{2}\right) x^{2} .
$$

La ecuación (13) se puede expresar como una ecuación cuártica en $x$ de la siguiente forma:

$$
\begin{gathered}
x^{4}+\frac{4 L^{2} \cos 4 \theta_{1}}{a^{2}} x^{3}-\frac{a^{4}-4 L^{4}+\left(a^{4}+2 L^{4}-4 a^{2} L^{2} \cos 2 \theta_{1}\right) \cos 4 \theta_{1}}{a^{4}} x^{2} \\
-\frac{4\left(L^{3}-a^{2} L \cos 2 \theta_{1}\right)}{a^{6}} x+\frac{\left(L^{2}-a^{2} \cos 2 \theta_{1}\right)^{4}}{a^{8}}=0
\end{gathered}
$$

Las cuatro raíces de (14) pueden calcularse analíticamente usando la solución de Ferrari [3]. De esas cuatro raíces, desecharemos las soluciones complejas. Además, de acuerdo con la figura 2, escogeremos la raíz que cumpla $\theta_{2}=1 / 2 \cos ^{-1} x<\theta_{1}$.

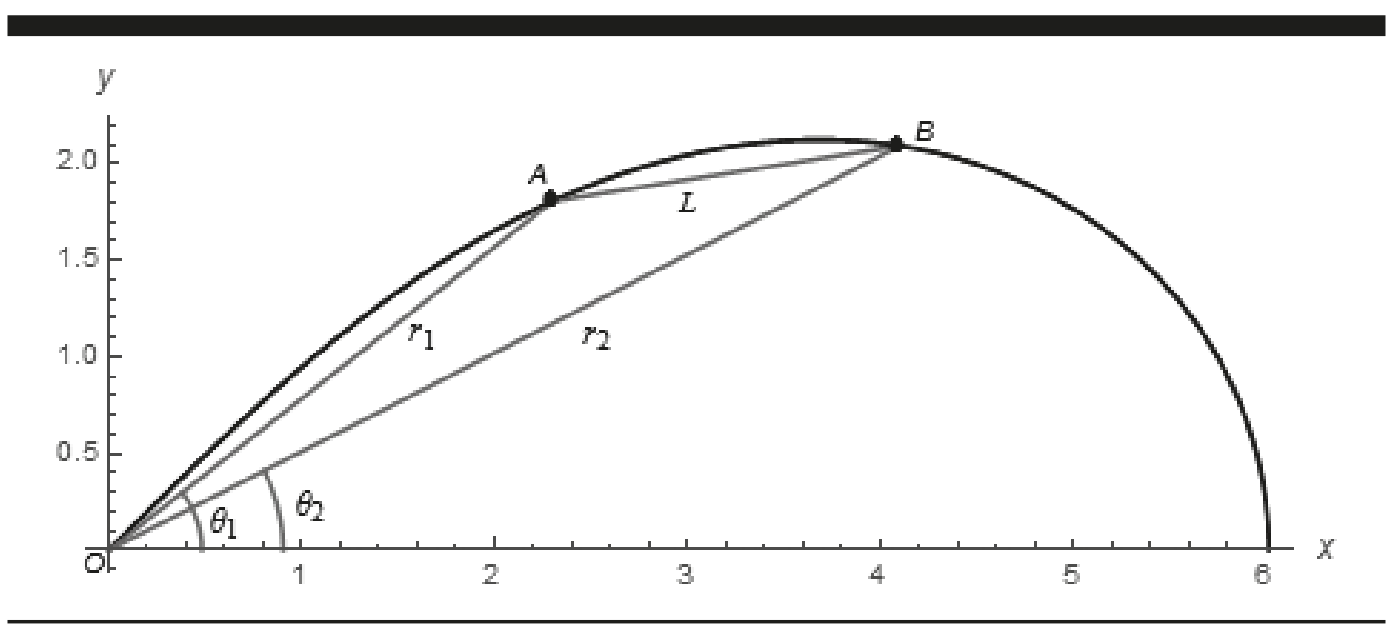

Fig. 2. Esquema para el cálculo de la posición de cabecera del vagón (punto B).

Una vez hallado el ángulo polar $\theta_{2}$ de la posición de la cabecera del vagón, podemos hallar sus coordenadas cartesianas $\left(x_{B}, y_{B}\right)$ con $(6)$. 


\section{CÁLCULO DE LA FLECHA}

Una vez establecida la posición del vagón sobre la vía (puntos $A$ y $B$ en la figura 3), hemos de calcular la flecha $f=C T$ entre la cuerda $A B$ y la curva. Para ello, hemos de determinar, en primer lugar, el punto de tangencia $T=\left(x_{T}, y_{T}\right)$. Si la pendiente de la cuerda es

$$
m=\tan \alpha=\frac{y_{B}-y_{A}}{x_{B}-x_{A}},
$$

el punto de tangencia $T$ satisface la ecuación $d y / d x=m$, que según (10) se traduce en resolver la siguiente ecuación $\forall t=t_{T}$,

$$
\left.\frac{1-3 t^{2}-3 t^{4}+t^{6}}{1+3 t^{2}-3 t^{4}-t^{6}}\right|_{t=t_{T}}=m .
$$

De este modo, según (9), las coordenadas del punto de tangencia vendrán dadas por $x_{T}=x\left(t_{T}\right)$ e $y_{T}$ $=y\left(t_{T}\right)$. Asimismo, cabe destacar que $\forall t \in[0,1], m \leq 1$.

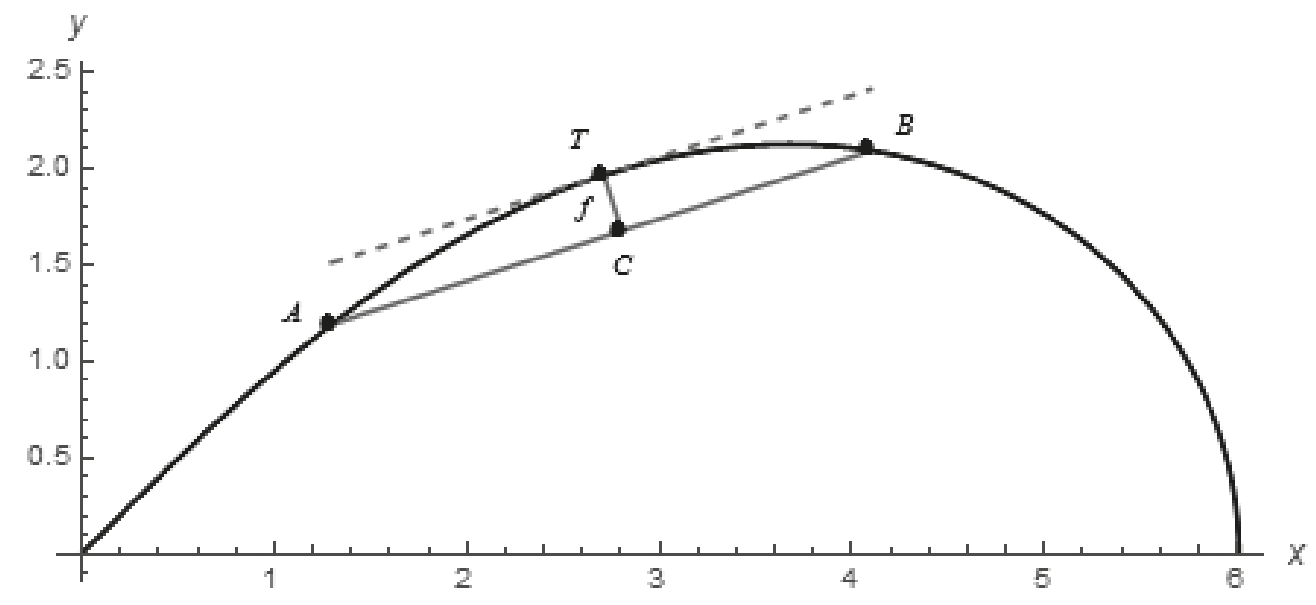

Fig 3. Esquema del cálculo de la flecha.

\section{Resolución del punto de tangencia}

A partir de (16), obtenemos la siguiente ecuación bicúbica:

$$
(1+m) t^{6}+3(m-1) t^{4}-3(m+1) t^{2}+m-1=0 .
$$


Con el cambio $z=t^{2}$, obtenemos:

$$
\left\{\begin{array}{cc}
z^{3}+3 \frac{m-1}{m+1} z^{2}-3 z+\frac{1-m}{1+m}=0, & m \neq-1, \\
-6 z^{2}+2=0, & m=1 .
\end{array}\right.
$$

Por tanto, cuando $m=-1, t_{T}=3^{-1 / 4}$. En el caso $m \neq-1$, tenemos que resolver una ecuación cúbica de con los coeficientes:

$$
a=3 \frac{m-1}{m+1}, \quad b=-3, \quad c=\frac{1-m}{1+m} .
$$

De acuerdo con [3], como los siguientes parámetros son positivos:

$$
\begin{aligned}
& P=\frac{a^{2}-3 b}{9}=\frac{2\left(m^{2}+1\right)}{(m+1)^{2}}>0, \\
& D=P^{3}-Q^{2}=\frac{4\left(m^{2}+1\right)^{2}}{(m+1)^{4}}>0
\end{aligned}
$$

donde

$$
Q=\frac{a b}{6}-\frac{c}{2}-\frac{a^{3}}{27}=-2 \frac{\left(m^{2}+1\right)(m-1)}{(m+1)^{3}}
$$

resulta que la ecuación cúbica dada en (18) tiene todas las raíces reales y se pueden expresar como,

$$
z_{k}=-\frac{a}{3}+2 \sqrt{P} \cos \left(\frac{\cos ^{-1}\left(Q P^{-3 / 2}\right)+2 \pi k}{3}\right), \quad k=0, \pm 1
$$

En nuestro caso, tenemos que

$$
z_{k}=\frac{1-m}{1+m}+2 \frac{\sqrt{2\left(1+m^{2}\right)}}{|1+m|} \cos \left(\frac{1}{3}\left[\cos ^{-1}\left(\frac{(1-m) \operatorname{sgn}(1+m)}{\sqrt{2\left(1+m^{2}\right)}}\right)+2 \pi k\right]\right)
$$

Como $z=t^{2}$ y $t \in[0,1]$, entonces $z \in[0,1]$. De este modo, hemos de escoger $k$, tal que $z_{k} \in[0,1]$ y calcular $t_{T}=z_{k}^{1 / 2}$. 


\section{Resolución del cálculo de la flecha}

Una vez hallado el punto de tangencia $T=\left(x_{T}, y_{T}\right)$, hemos de calcular el punto $C=\left(x_{C}, y_{C}\right)$ de la cuerda (figura 3). Para ello, tenemos que resolver el sistema de ecuaciones lineal correspondiente a la cuerda,

$$
y-y_{A}=m\left(x-x_{A}\right)
$$

y a la ecuación perpendicular a la cuerda que pasa por el punto de tangencia $T$,

$$
y-y_{T}=\frac{-1}{m}\left(x-x_{T}\right)
$$

de tal manera que

$$
\left\{\begin{array}{l}
x_{C}=\frac{m\left(y_{T}-y_{A}\right)+m^{2} x_{A}+x_{T}}{1+m^{2}} \\
y_{C}=y_{T}+\frac{x_{T}-x_{C}}{m}
\end{array}\right.
$$

La flecha $f$ es precisamente la distancia $C T$ :

$$
\begin{aligned}
f & =\sqrt{\left(x_{T}-x_{C}\right)^{2}+\left(y_{T}-y_{C}\right)^{2}} \\
& =\frac{\left|y_{T}-y_{A}+m\left(x_{A}-x_{T}\right)\right|}{\sqrt{1+m^{2}}} .
\end{aligned}
$$

En la figura 4 podemos apreciar cómo varía la flecha $f$ según la posición de la cola del vagón $x_{a}$ para distintos valores del parámetro $a$ de la lemniscata y una longitud $L=1,5$ para el vagón. 


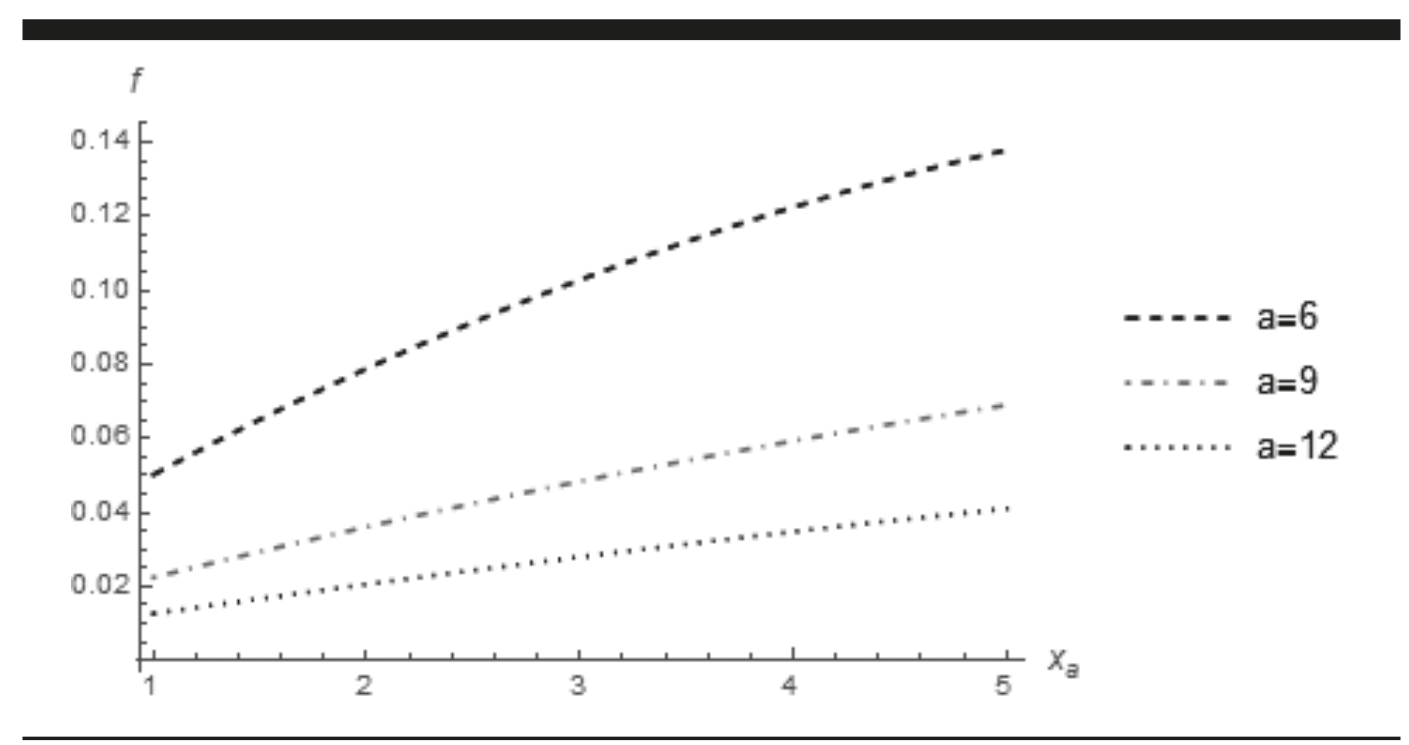

Fig. 4. Variación de la flecha para distintos valores de a con $\mathrm{L}=1,5$.

\section{CONCLUSIONES}

En este artículo hemos obtenido unas expresiones analíticas para calcular la flecha de una cuerda en una curva lemniscata. Dado que las curvas lemniscatas se utilizan como trazados de transferencia en las vías ferroviarias, estas expresiones son útiles para determinar si dichos trazados respetan la normativa de seguridad para una longitud dada de vagón de tren. Las expresiones analíticas deducidas en este artículo se encuentran disponibles en un fichero de Mathematica ${ }^{\mathrm{r}}$, en la dirección: https://bit. ly/2OttbLa.

\section{AGRADECIMIENTOS}

El autor desea agradecer a D. Jose Antonio Ruiz Calero el planteamiento de este problema.

\section{REFERENCIAS BIBLIOGRÁFICAS}

[1] Blanch L, Checa E, Marín J. Una aproximación a la curva de transición Clotoide vista desde Mathematica. Modelling in Science Education and Learning. 2013;6:105-19.

[2] Spiegel MR. Handbook of Mathematical Formulas. New York, USA: Schaum-McGraw Hill, 1968.

[3] Oldham KB, Myland J, Spanier J. An atlas of functions: with equator, the atlas function calculator. Springer Science \& Business Media, 2010. 
